\title{
Lung abscess in immunocompromised
}

We present the case of a 44-year-old man, construction worker, hypertensive, smoker, with rheumatoid arthritis under prednisolone $10 \mathrm{mg} /$ day for 10 months. Gingival crevice disease. The patient was referred to the Emergency Department after 5 days of right chest pain with pleuritic features, cough, purulent sputum and dyspnea grade 3 mMRC, along with several weeks of indolent symptoms (anorexia, weight loss and night sweats). Polypneic with abdominal breathing and hypotensive. Initial blood tests showed an increase in inflammatory markers, hypoxemic respiratory failure and hyperlactacidemia. A thick walls cavitation, with an hydroaereal level was present in the chest X-ray (Fig 1). Thoracic computerized tomography (CT) shows a voluminous apparently loculated image occupying almost all of the right lung field associated with homolateral pleural effusion; a nodular image of about $28 \mathrm{~mm}$ with hydroaereal content located to the lower left lobe and at least two other identical smaller images in the same lobe (Fig 2). Diagnosed with lung abscess (LA), he was admitted to Intensive Care in septic shock under invasive ventilation and aminergic support, starting empirically piperacillin/tazobactam. The evaluation of pleural effusion (PE) reveals empyema fluid with an increased ADA of 158 IU/L. Tube thoracostomy with 3 chest tubes was performed. No structural changes in bronchofibroscopy (FB). PE culture identifies $\mathrm{H}$. influenzae, S. parasanguinis and S. salivarius, allowing targeted therapy with clindamycin, fulfilling 35 days. Sterile blood cultures. PE cytology without malignancy. Tuberculosis excluded (PE and sputum with negative acidfast bacilli (AFB) smear and culture; negative polymerasechain-reaction assay for M. tuberculosis on bronchoalveolar lavage (BAL) and PE). Follow-up at 3 months with sterile BAL cultures and negative AFB BAL smear. CT improvement, with only residual changes, 6 months later.

LA consists of pulmonary parenchymal necrosis caused by microbial infection. Primary in $80 \%$ of cases. Inoculum aspiration from gingival crevice, poor dental hygiene are the main causes ${ }^{1}$. It as been noticed the close relationship with bacteria found in the oral cavity and gingival sulci. Steroids and smoking are important risk factors ${ }^{2,3}$. Cough, sputum, fever and systemic manifestations are a common presentation. Typically diagnosed with chest X-ray, anatomical definition is improved with CT. Despite difficult cultural isolation due to frequent contamination, Gram and sputum cultures are indicated as well as blood and PE cultures. FB is important in obtaining a cultural sample and exclusion of another etiology. Bacillary disease should be excluded. Anaerobes are present in $60-80 \%$ of cases, with frequently associated oral Streptococcus, $90 \%$ are polymicrobial ${ }^{4}$. Our patient's agents were facultative oral anaerobes. Etiological agents and associated comorbidities are essential in defining therapeutic strategy $y^{5,6}$.
Figure 1. Chest X-ray

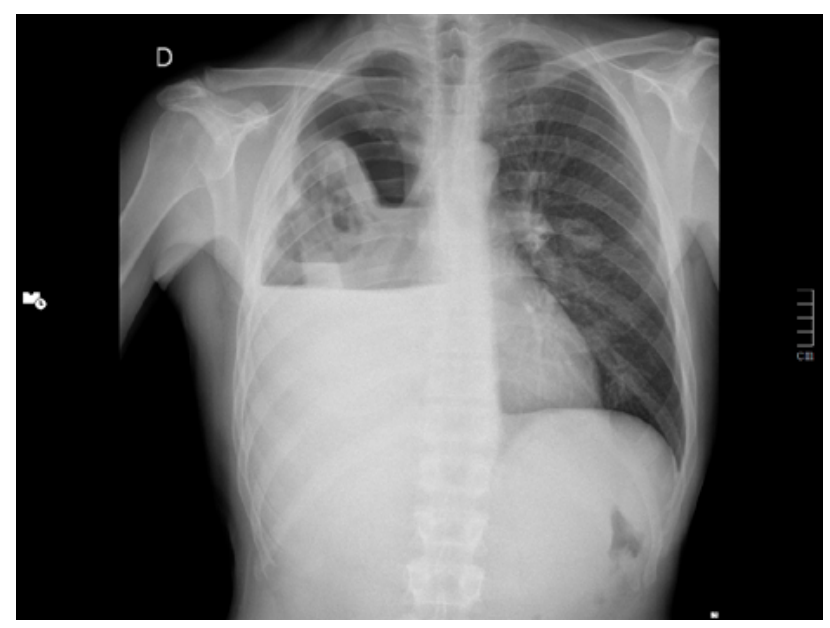

Figure 2. Thoracic computerized tomography

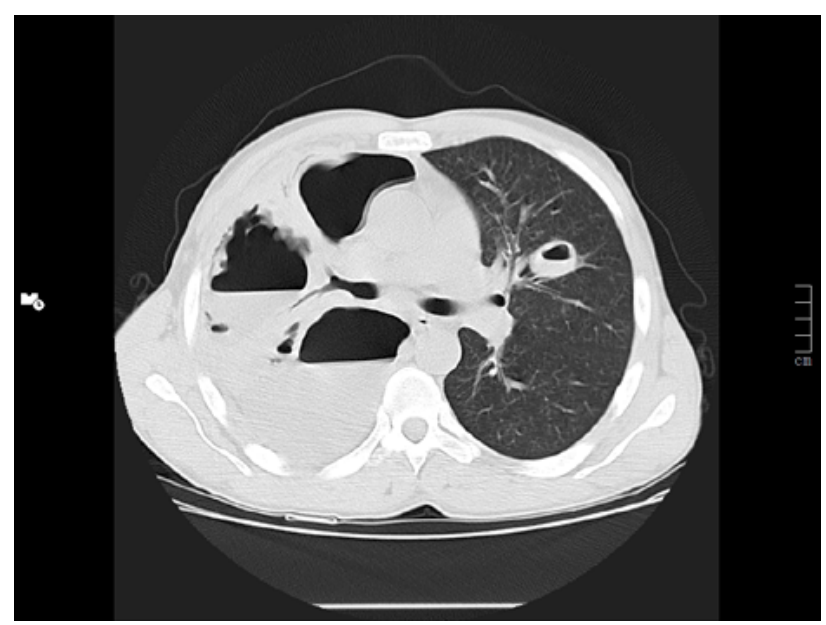

Antibiotics are usually maintained until radiological resolution or stable residual lesion, this may require several months and can be performed with an outpatient oral regimen. Bronchial obstruction, diameter $>6 \mathrm{~cm}$, neoplasia, immunodepression and resistant microorganisms are predictors of poor therapeutic response ${ }^{7,8}, 90-95 \%$ of primary abscesses can cure ${ }^{9,10}$. Mortality rate in the immunocompromised may exceed $75 \%{ }^{11}$.

In summary, the authors, regarding an exuberant radiological presentation, intend to emphasize the relevance of microbiological isolation in targeting antimicrobial options, enabling faster outpatient treatment, avoiding prolonged hospitalization and complications inherent to it. 


\section{ACKNOWLEDGEMENTS}

The authors gratefully acknowledge to Dr. Ricardo Meireles (Internal Medicine Department, Centro Hospitalar do Tâmega e Sousa, Penafiel, Portugal) and the Pulmonology Department (Centro Hospitalar do Tâmega e Sousa, Penafiel, Portugal)

\section{BIBLIOGRAPHY}

1. Puligandla PS, Laberge JM. Respiratory infections: pneumonia, lung abscess, and empyema. Semin Pediatr Surg 2008;17:42-52.

2. Gonçalves AM, Menezes Falcão L, Ravara L. Pulmonary abcess, a revision. Rev Port Pneumol 2008;14:141-9.

3. Magalhães L, Valadares D, Oliveira JR, et al. Lung abscesses: review of 60 cases. Rev Port Pneumol

4. Madhani K, McGrath E, Guglani L. A 10-year retrospective review of pediatric lung abscesses from a single center. Ann Thorac Med 2016; 11:191.

5. Goldstein EJ, Citron DM, Warren Y, et al. In vitro activity of gemifloxacin (SB 265805) against anaerobes. Antimicrob Agents Chemother 1999; 43:2231.

6. Levison ME, Mangura CT, Lorber B, et al. Clindamycin compared with penicillin for the treatment of anaerobic lung abscess. Ann Intern Med 1983; 98:466.

7. Clinical conferences at the Johns Hopkins Hospital: lung abscess. Johns Hopkins Med J 1982; 150:141.

8. Hirshberg B, Sklair-Levi M, Nir-Paz R, et al. Factors predicting mortality of patients with lung abscess. Chest 1999; 115:746.

9. Bartlett JG. Lung abscess and necrotizing pneumonia. In: Infectious Diseases, Gorbach SL, Bartlett JG, Blacklow NR (Eds), W.B. Saunders, Philadelphia 1992.

10. Bartlett JG. Treatment of anaerobic pleuropulmonary infections. Ann Intern Med 1975; 83:376.

11. Pohlson EC, McNamara JJ, Char C, Kurata L. Lung abscess: a changing pattern of the disease. Am J Surg 1985; 150:97.

\section{DIAGNOSIS \\ Lung abscess in immunocompromised}

Teresa Dias Moreira, Lígia Rodrigues dos Santos

Internal Medicine Department, Centro Hospitalar do Tâmega e Sousa, Penafiel, Portugal.

Correspondencia: teresa_margaridam@hotmail.com

Cómo citar este artículo: Dias Moreira T, Rodrigues dos Santos L

Lung abscess in immunocompromised. Galicia Clin 2020; 81 (2): 57-58

Recibido: 22/12/2018; Aceptado: 23/01/2019 // http://doi.org/10.22546/56/1868 